\title{
Comparison of calcium-channel blockers for long- term clinical outcomes in patients with vasospastic angina
}

\author{
Sung Eun Kim ${ }^{1}$, Sang-Ho Jo², Seung Hwan $\mathrm{Han}^{3}$, Kwan Yong Lee ${ }^{4}$, Sung Ho Her ${ }^{5}$, Min-Ho Lee ${ }^{6}$, \\ Won-Woo $\mathrm{Seo}^{1}$, Seong-Sik $\mathrm{Cho}^{7}$, and Sang Hong Baek ${ }^{8}$
}

\begin{abstract}
${ }^{1}$ Department of Cardiovascular Medicine, Hallym University Kangdong Sacred Heart Hospital, Seoul; ${ }^{2}$ Division of Cardiology, Department of Internal Medicine, Hallym University Sacred Heart Hospital, Anyang; ${ }^{3}$ Department of Cardiovascular Medicine, Gachon University Gil Medical Center, Incheon; ${ }^{4}$ Department of Cardiovascular Medicine, Incheon St. Mary's Hospital, College of Medicine, The Catholic University of Korea, Incheon; ${ }^{5}$ Department of Cardiovascular Medicine, Daejeon St. Mary's Hospital, College of Medicine, The Catholic University of Korea, Daejeon; ${ }^{6}$ Department of Cardiovascular Medicine, Soonchunhyang University Seoul Hospital, Seoul; ${ }^{7}$ Department of Epidemiology and Occupational Medicine, Dong-A University College of Medicine, Busan; ${ }^{8}$ Department of Cardiovascular Medicine, Seoul St. Mary's Hospital, College of Medicine, The Catholic University of Korea, Seoul, Korea
\end{abstract}

Received: September 16, 2019 Revised : November 10, 2019 Accepted: December 19, 2019
Background/Aims: Calcium channel blockers (CCBs) are the most widely prescribed medication for patients with vasospastic angina (VA). However, few studies have compared the prognosis of VA patients who are prescribed different CCBs. Methods: We enrolled 2,960 patients who received provocation test prospectively in 11 university hospitals in Korea. We divided 1,586 patients received four major CCBs into two groups: a first generation CCB (diltiazem and nifedipine) group and a second generation CCB (amlodipine and benidipine) group. Primary outcome was time to events of composite of death from any cause, acute coronary syndrome (ACS) and symptomatic arrhythmia during 3-year follow-up. We also compared the effect of each CCB on the control of angina symptoms.

Results: There was no difference of the primary outcome among the two groups with a cumulative incidence rate of 5.4\%, $2.9 \%$, and a person-month incidence rate of 2.33 and 1.26, respectively (hazard ratio [HR], 0.54; 95\% confidence interval [CI], 0.25 to $1.17 ; p=0.120$, as reference with the 1st generation CCBs). The incidence of ACS was significantly lower in 2nd generation CCBs group with a person-month incidence rate of 1.66 vs. 0.35 (HR, $0.22 ; 95 \%$ CI, 0.05 to $0.89 ; p=0.034$ ). Use of benidipine showed a significant better control of angina symptom compared with diltiazem for 3 years (odds ratio, $0.17 ; 95 \% \mathrm{CI}, 0.09$ to $0.32 ; p<0.0001$ at 3 rd year).

Conclusions: The first and second generation CCB groups did not differ in terms of composite outcome occurrence. However, the ACS incidence rate was significantly lower in the users of the 2nd generation CCBs.

Keywords: Coronary vasospasm; Calcium channel blockers; Patient outcome assessment

Correspondence to Sang-Ho Jo, M.D.

Division of Cardiology, Department of Internal Medicine, Hallym University Sacred Heart Hospital, 22 Gwanpyeong-ro 17obeon-gil, Dongan-gu, Anyang 14068, Korea Tel: +82-31-380-3722, Fax: +82-31-386-2269, E-mail: sophi5neo@gmail.com https://orcid.org/0000-0002-2063-1542 


\section{INTRODUCTION}

Vasospastic angina (VA) is highly prevalent in Korea and Japan [1-4]. Although patients with VA are known to have favorable long-term prognosis [5], critical patients who present with acute coronary syndrome (ACS) and cardiac arrest have been reported $[6,7]$. Therefore some investigators argue that the prognosis of VA is varied and the treatment ought to be more refined and tailored to match a patients specific needs [8].

Calcium channel blockers (CCBs) are usually prescribed to VA patients in an effort to prevent angina attacks with coronary vasospasm, and reduce the occurrence of accompanying sudden cardiovascular death and other cardiac events $[4,9,10]$. The first generation of CCBs like nifedipine, verapamil and diltiazem, which were introduced in 1980s, have been validated for the control of angina in patients with VA [11-13]. The new generation of CCBs including amlodipine, nicardipine, and benidipine were validated in 1990 s [14,15]. However, few studies have compared the prognostic and symptom relieving effects of first and second generation CCBs.

In the present study, we compared the long-term clinical outcomes and angina relieving properties of CCBs in patients with VA confirmed by coronary angiography (CAG) and an ergonovine (EG) provocation test from a large prospective multicenter cohort in Korea.

\section{METHODS}

The variant angina-Korea (VA-Korea) registry, which is a nation-wide prospective multicenter registry, enrolled patients with chest pain indicative of VA who had received CAG and an EG provocation test. Adults aged 18 or over were considered for enrollment in the study. Patients who had a normal or minimal $(<50 \%$ luminal diameter narrowing) coronary atherosclerotic stenosis at the baseline in CAG could be registered. Patients with malignancy, end stage renal disease requiring dialysis, inflammatory disease, or catheter-induce spasm at baseline CAG were excluded from the study.

The study protocol was approved by the Institutional Review Board of each participating hospital(Hallym IRB No. 2010-Ioo7) and all patients gave written informed consent. All procedures performed in the studies in- volving human participants were in accordance with the ethical standards of the institutional and/or national research committee, and with the 1964 Helsinki Declaration and its later amendments or comparable ethical standards.

A total of 2,960 patients were registered consecutively from May 2010 to June 2015 in 11 tertiary hospitals in Korea with high volume CAG and percutaneous coronary intervention procedures. Among them, 1,892 patients were judged to have positive (definite, 680) and intermediate $(1,212)$ results in their provocation tests. Fifty-four patients were lost in the follow-up; thus, finally 1,838 patients with follow-up data were further investigated. Among them, 1,586 patients were prescribed representative CCBs including diltiazem, nifedipine, amlodipine, and benidipine.

Patients with positive results in the EG provocation test and with a spontaneous spasm received medical treatments including CCBs and other vasodilators during the follow-up. The grade of angina was evaluated at 1-month, 1-, 2-, and 3-year follow-up period. Patients were divided into two groups: the patients treated with the 1st generation CCBs (diltiazem, nifedipine users) and patients treated with the and generation CCBs (amlodipine and benidipine). We followed up the clinical events recorded in the study participants for 3 years.

The primary endpoint is the composite of death from any cause, ACS, and a new-onset of symptomatic arrhythmia during the 3-year follow-up. ACS was defined as a recurrent or continuous ischemic chest pain lasting more than 20 minutes with ischemic symptoms detected by electrocardiographic (ECG) changes and/or elevation of cardiac markers including myocardial infarction (MI). The ischemic ECG changes used as markers of ischemia were an elevation of the ST of $0.1 \mathrm{mV}$ or more, a depression of the ST of $0.1 \mathrm{mV}$ or more, a T-wave inversion, or the occurrence of a left bundle branch block recorded in at least two contiguous leads on the 12-lead ECG. We defined a new-onset symptomatic arrhythmia as the first occurrence of either atrial or ventricular tachycardia/ fibrillation, symptomatic premature beats, sick-sinus rhythm, or atrioventricular block. The ECG was routinely checked during the regular follow-up and emergent visits to the out-patient clinic or emergency department of the hospital. Holter monitoring for the first 24-hour was performed in patients with suspected arrhythmic 
symptoms. The frequency of angina after discharge was presented according to the prior classification; grade I: near-daily attacks; II: $\geq 4$ attacks/month; III: $\geq 1$ but $<4$ attacks/month; and IV: < 1 attack/month [16].

Emergency room revisits due to the occurrence of the primary endpoint or of any discomfort were reported as soon as possible. Individual adverse events were analyzed as a secondary endpoint. All the adverse events of interest were confirmed through source document review, including the medical records as well as telephone interviews, and were adjudicated by the Local Events Committee of Seoul St. Mary's Hospital. All the patients received baseline CAG by routine methods after the cessation of vasoactive drugs like CCBs and nitrate for 48 hours prior to the CAG. All participating hospitals used the same protocol of EG provocation like EG administration route and serial doses.

After the physicians' confirmed that there was no significant atherosclerotic coronary narrowing from baseline CAG, the EG provocation test was performed starting with the right coronary artery (RCA). If the RCA was intact, the left coronary artery (LCA) was tested. The EG was mixed with saline and administered by an intracoronary (IC) bolus injection over a 2 to 3 minutes. If no narrowing was found after the 1st dose of EG on the RCA angiogram, the dose was escalated from 10 (E1) to $2 \mathrm{O}\left(\mathrm{E}_{2}\right)$ and $4 \mathrm{O} \mu \mathrm{g}\left(\mathrm{E}_{3}\right)$ sequentially. CAG was performed 1 to 2 minutes after the completion of the EG injection in the same projection compared to the vessel diameter of the baseline RCA.

If a coronary spasm was not provoked after testing the RCA, an EG provocation on left anterior descending artery followed with incremental doses of 20 (E1), 40 (E2), and $60 \mu \mathrm{g}\left(\mathrm{E}_{3}\right)$ in the same manner. Once spasm was provoked, IC nitroglycerine of $200 \mu \mathrm{g}$ bolus was injected.

After the provocation test, irrespective of the test result, IC nitrate $(200 \mu \mathrm{g})$ was injected and the response was observed. We followed the provocation methods from the Japanese Circulation Society guideline for the diagnosis and treatment of patients with VA.

The CAG result was analyzed by investigating all the segments of each inter coronary artery. The angiographic findings were analyzed on-line or off-line by a dedicated quantitative CAG program (Syngo X Workplace version VA6oC from syngo QCA system, Siemens AG, Munich, Germany) or through manual assessment by the investigators in each hospital who were not involved in the study. In addition, investigators in the core laboratory of Seoul St. Mary's Hospital in South Korea, confirmed in blinded angiographic data off-line by visual assessment. Meaningful atherosclerosis in each coronary artery was defined as a luminal diameter narrowing of $\geq 50 \%$.

Definite (positive) VA was defined as a total (100\%) or subtotal (> 90\% luminal diameter narrowing) occlusion of the index coronary artery accompanied with ischemic symptoms and/or ECG changes. An ischemic ECG change was defined as an ST segment elevation or depression of $>0.1 \mathrm{mV}$ or a negative U-wave in at least two contiguous leads [16]. An intermediate result was defined as patients with $50 \%$ to $90 \%$ luminal narrowing with or without ischemic symptoms and/or ECG changes. We define negative results as both an LCA and RCA EG provocation test with $<50 \%$ luminal narrowing without ischemic symptoms or ECG changes. Besides the overall statistical analysis, we made an additional analysis in patients with definite spasm. Continuous variables were expressed as the mean and standard deviation, and mean differences between groups were estimated by the analysis of variance (ANOVA). Categorical variables were demonstrated with the absolute number and with percentages. Each incidence rate and $95 \%$ confidence interval (CI) was demonstrated according to the CCB treatment. The event rate per 1,000 person month was displayed for estimating the incidence rate of the primary endpoint, ACS, arrhythmia, and all cause death, and Kaplan-Meier curves were presented to estimate the survival. The log-rank test was conducted to compare the survival among the different groups. In addition, the Cox proportional hazard regressions were conducted to estimate the survival difference among groups using the hazard ratio (HR) with a $95 \%$ CI. Schoenfeld's partial residuals were utilized to test for proportionality. Subgroup analysis was conducted to compare the incidence of the primary outcomes across different clinical conditions that may influence the prognosis, and an interaction analysis was conducted to examine the heterogeneity. Ordered logistic regression analysis was employed for comparing the severity of angina symptoms between the different CCBs. A p value of < 0.05 , was considered statistically significant. Statistical analysis was carried out using Stata version 13.1 (Stata Corp., College station, TX, USA). 
Table 1. Demographic and clinical characteristics of study participants

\begin{tabular}{|c|c|c|c|c|}
\hline Characteristic & $\begin{array}{c}\text { Total } \\
(\mathrm{n}=1,586)\end{array}$ & $\begin{array}{l}\text { 1st generation } \mathrm{CCBs}^{\mathrm{a}} \\
(\mathrm{n}=1,345)\end{array}$ & $\begin{array}{l}\text { 2nd generation } \mathrm{CCBs}^{\mathrm{b}} \\
\qquad(\mathrm{n}=24 \mathrm{1})\end{array}$ & $p$ value \\
\hline Age, yr & $55.10 \pm 11.04$ & $54.92 \pm 11.09$ & $56.12 \pm 10.70$ & 0.119 \\
\hline $\mathrm{BMI}, \mathrm{kg} / \mathrm{m}^{2}$ & $28.39 \pm 89.56$ & $29.05 \pm 97.54$ & $24.87 \pm 3.13$ & 0.130 \\
\hline $\mathrm{SBP}, \mathrm{mmHg}$ & $127.39 \pm 33.11$ & $127.10 \pm 35.12$ & $129.01 \pm 18.38$ & 0.210 \\
\hline DBP, mmHg & $77.25 \pm 12.33$ & $77.09 \pm 12.38$ & $78.17 \pm 12.03$ & 0.209 \\
\hline $\mathrm{TC}, \mathrm{mg} / \mathrm{dL}$ & $175.17 \pm 36.40$ & $175.60 \pm 35.95$ & $172.82 \pm 38.86$ & 0.301 \\
\hline $\mathrm{TG}, \mathrm{mg} / \mathrm{dL}$ & $144.18 \pm 104.84$ & $143.84 \pm 104.71$ & $146.10 \pm 105.81$ & 0.775 \\
\hline HDL-C, mg/dL & $46.77 \pm 12.74$ & $46.82 \pm 12.99$ & $46.53 \pm 11.31$ & 0.735 \\
\hline LDL-C, mg/dL & $104.15 \pm 31.79$ & $104.72 \pm 31.54$ & $101.08 \pm 33.00$ & 0.130 \\
\hline hsCRP, mg/dL & $0.95 \pm 7.28$ & $1.07 \pm 8.00$ & $0.37 \pm 0.84$ & 0.008 \\
\hline $\mathrm{CKMB}, \mathrm{ng} / \mathrm{mL}$ & $6.27 \pm 25 \cdot 34$ & $6.57 \pm 26.76$ & $4.13 \pm 10.28$ & 0.047 \\
\hline Troponin-I, ng/mL & $0.61 \pm 4.74$ & $0.67 \pm 5.04$ & $0.21 \pm 1.57$ & 0.059 \\
\hline LVEF, \% & $64.49 \pm 6.42$ & $64.58 \pm 6.27$ & $64.02 \pm 7.15$ & 0.282 \\
\hline Male sex & $996(62.80)$ & $826(61.41)$ & $170(70.54)$ & 0.007 \\
\hline Smoking & $446(28.46)$ & $389(29 \cdot 31)$ & $57(23.75)$ & 0.079 \\
\hline HTN & $601(37.92)$ & $480(35.71)$ & $121(50.21)$ & $<0.001$ \\
\hline $\mathrm{DM}$ & $141(8.90)$ & $124(9.23)$ & $17(7.08)$ & 0.283 \\
\hline Dyslipidemia & $274(17.31)$ & $233(17 \cdot 35)$ & $41(17.08)$ & 0.920 \\
\hline CHD & $194(12.25)$ & $154(11.46)$ & $40(16.67)$ & 0.023 \\
\hline PCI & $35(2.21)$ & $31(2.30)$ & $4(1.66)$ & 0.530 \\
\hline Definite spasm & $621(39.16)$ & $531(39 \cdot 48)$ & $90(37 \cdot 34)$ & 0.532 \\
\hline Atherosclerosis > $>0 \%$ & $122(7.69)$ & $106(7.88)$ & $16(6.64)$ & 0.505 \\
\hline
\end{tabular}

Values are presented as mean \pm SD or number (\%). $p$ values were calculated by $t$ test for continuous variables and chi-square test for categorical variables.

CCB, calcium channel blocker; BMI, body mass index; SBP, systolic blood pressure; DBP, diastolic blood pressure; TC, total cholesterol; TG, triglyceride; HDL-C, high density lipoprotein cholesterol; LDL-C, low density lipoprotein cholesterol; hsCRP, high-sensitive C-reactive protein; CKMB, creatinine kinase-MB; LVEF, left ventricular ejection fraction; HTN, hypertension; DM, diabetes mellitus; CHD, coronary heart disease; PCI, percutaneous coronary intervention.

${ }^{\mathrm{a}}$ 1st-generation CCBs indicate diltiazem and nifedipine.

$\mathrm{b}_{\text {2nd-generation CCBs indicate amlodipine and benidipine. }}$

\section{RESULTS}

We enrolled 2,960 patients from 11 hospitals in Korea. Among them, 1,838 patients experienced definite/intermediate spasm, 1,675 received CCBs (90.2\%), and 1,586 patients were prescribed with one of the four major CCBs—diltiazem, nifedipine, amlodipine, and benidipine. These patients entered the final analysis.

The mean follow-up period was $22.63 \pm 16.21$ months (median, 23.90 [interquartile range, 9.56 to 23.90]). We divided the patients into two groups, patients receiving the 1st generation CCBs (diltiazem and nifedipine) and patients receiving the 2 nd generation CCBs (amlodipine and benidipine).

The baseline characteristics of the patients are shown in Table 1. The mean age ranged from 53.7 to 57 years. Diltiazem was the most widely prescribed drug (79.0\%) followed by amlodipine (11.1\%), nifedipine (5.8\%), and benidipine (4.1\%) (Supplementary Table 1). The male sex and CHD history were more common in the patients prescribed with 2nd generation CCBs (Table 1).

\section{Comparison of the 1st and 2nd generation CCBs}

We compared the primary outcome defined as the com- 
Table 2. Incidence rates and HRs for primary composite outcomes and each outcome between 1st and 2nd generation CCBs

\begin{tabular}{|c|c|c|c|c|c|c|c|c|c|}
\hline Variable & No. & Event (\%) & Person-month & Incidence rate & $95 \% \mathrm{CI}$ & $p$ value $^{\mathrm{a}}$ & $\mathrm{HR}^{\mathrm{b}}$ & $95 \% \mathrm{CI}$ & $p$ value $^{b}$ \\
\hline Composite outcome & & & & & & 0.110 & & & \\
\hline 1st generation $\mathrm{CCBs}^{\mathrm{c}}$ & 1,345 & $72(5.4)$ & 30,842 & 2.33 & $1.85-2.94$ & & Reference & & \\
\hline 2nd generation $\mathrm{CCBs}^{\mathrm{d}}$ & 241 & $7(2.9)$ & 5,570 & 1.26 & $0.60-2.64$ & & 0.54 & $0.25^{-1.17}$ & 0.120 \\
\hline Arrhythmia & & & & & & 0.703 & & & \\
\hline 1st generation CCBs & 1,345 & $19(1.4)$ & 32,483 & 0.58 & $0.37-0.92$ & & Reference & & \\
\hline 2nd generation CCBs & 241 & $4(1.7)$ & 5,639 & 0.71 & $0.27-1.89$ & & 1.23 & $0.42-3.63$ & 0.704 \\
\hline ACS & & & & & & 0.019 & & & \\
\hline 1st generation CCBs & 1,345 & $53(3.9)$ & 31,981 & 1.66 & $1.27-2.17$ & & Reference & & 0.034 \\
\hline 2nd generation CCBs & 241 & $2(0.8)$ & 5,700 & 0.35 & $0.09-1.40$ & & 0.22 & $0.05-0.89$ & \\
\hline \multicolumn{10}{|l|}{ Death } \\
\hline 1st generation CCBs & 1,345 & $4(0.3)$ & 32,873 & 0.12 & $0.05-0.32$ & & Reference & & \\
\hline 2nd generation CCBs & 241 & $1(0.4)$ & 5,742 & 0.17 & $0.02-1.24$ & & 1.31 & $0.15-11.79$ & 0.810 \\
\hline
\end{tabular}

HR, hazard ratio; CCB, calcium channel blocker; CI, confidence interval; ACS, acute coronary syndrome.

${ }^{a} p$ value by log rank test.

${ }^{\mathrm{b}} \mathrm{p}$ value by univariable Cox proportional hazard regression.

c1st-generation CCBs indicate diltiazem and nifedipine.

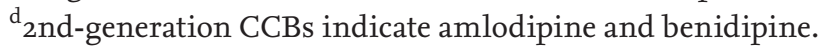

posite of total death, ACS, and new-onset symptomatic arrhythmia, between the 1st generation CCB group (diltiazem and nifedipine, $\mathrm{n}=1,345)$ and the and generation CCB group (amlodipine and benidipine, $\mathrm{n}=241$ ). There was no statistical difference between the two groups, with a crude incidence rate of $5.4 \%(72 / 1,345)$ and $2.9 \%$ (7/241), and an event rate of 2.33 (in 30,842 person-month), 1.26 (in 5,570 person-month) respectively (HR, $0.54 ; 95 \%$ CI, 0.25 to $1.17 ; p=0.120$, as reference of the 1st generation CCBs) (Table 2, Fig. 1). The individual event of death and symptomatic arrhythmia were not statistically different between the two groups (Table 2, Fig. 1).

However, with regard to ACS occurrence, the and generation CCB group showed a significantly lower event rate than the 1st generation CCB group, with an event rate of $0.8 \%$ vs. $3.9 \%$ (2nd vs. 1st generation respectively), and with a person-time incidence rate of $1.66(31,981$ person-month) vs. 0.35 (5,700 person-month) (HR, 0.22; $95 \%$ CI, 0.05 to $0.89 ; p=0.034$ ) (Table 2, Fig. 1).

Nonetheless, by multivariate cox regression analysis, the use of the 2nd generation CCBs was not an independent risk factor for the primary outcome (Table 3). In the subgroup analysis, there was no difference between the 1st and 2nd generation CCBs in the primary outcome occurrence (Table 4).
We also compared four different types of CCBs (Supplementary Tables 1 and 2). The primary outcome was not different among four CCBs groups (Supplementary Tables 1 and 2) in crude event rate and person-month incidence rate. By a Cox proportional hazard regression analysis, the individual groups of CCBs had similar efficacy as compared with the diltiazem group as a reference in the primary outcome and the primary outcome plus re-admission/emergency room visit (Supplementary Tables 3 and 4).

\section{Angina symptom relief}

Patients treated with benidipine had significantly better controlled angina symptoms as measured by the frequency of attack symptoms (grade I: near-daily attacks; II: $\geq 4$ attacks/month; III: $\geq 1$ but $<4$ attacks/month; and IV: $<1$ attack/month), as compared to patients treated with diltiazem after 1 year (adjusted odds ratio [OR], 0.43; $95 \% \mathrm{CI}, 0.23$ to $0.79 ; p=0.006$ ) both unadjusted and adjusted by ordered logistic regression analysis (Table 5). The beneficial effect persisted at the 2nd and 3 rd year ([OR, 0.20; 95\% CI, 0.10 to $0.41 ; p<0.0001$ at 2 nd year] and [OR, $0.19 ; 95 \%$ CI, 0.10 to $0.37 ; p<0.0001$ at 3 rd year]) (Table 5). 

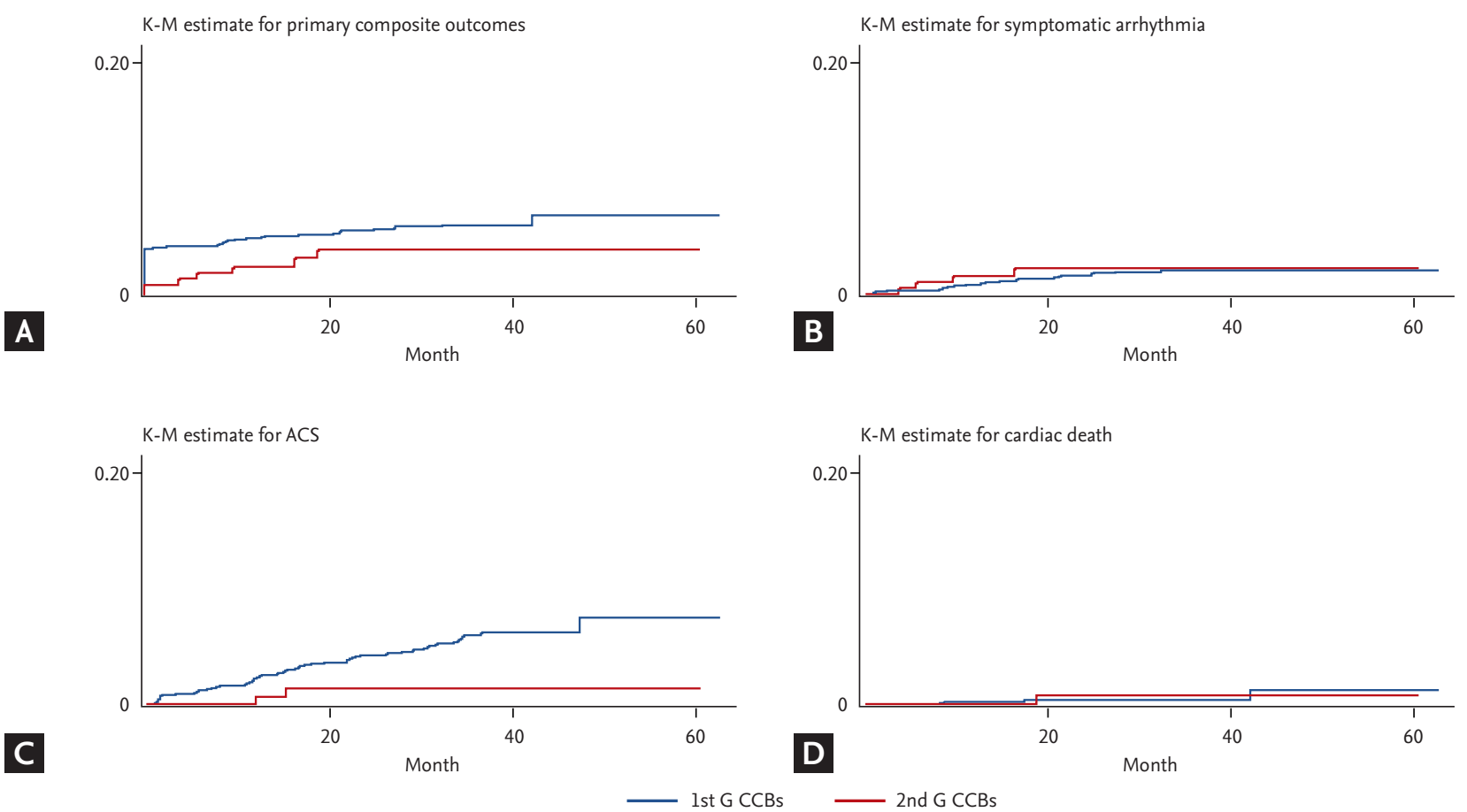

Figure 1. Primary and individual outcomes according to calcium channel blockers (CCBs) usage with Kaplan-Meier (K-M) survival curve. There was no significant difference in the incidence rate of primary outcomes between the 1st and 2nd generation CCB groups. (A) K-M estimate for primary outcomes, (B) K-M estimate for symptomatic arrhythmia, (C) K-M estimate for acute coronary syndrome, (D) K-M estimate for cardiac death.

Table 3. HR for the primary composite outcome by Cox regression including CCBs generation

\begin{tabular}{lccc}
\hline Variable & HR & $95 \%$ CI & p value \\
\hline Age & 1.00 & $0.98-1.02$ & 0.774 \\
Female sex & 0.86 & $0.50-1.49$ & 0.587 \\
\hline History of CHD & 1.69 & $0.95-3.00$ & 0.076 \\
Smoking & 1.25 & $0.72-2.15$ & 0.427 \\
\hline Alcohol drinking & 0.73 & $0.44-1.22$ & 0.235 \\
HTN & 0.78 & $0.48-1.28$ & 0.326 \\
\hline DM & 1.06 & $0.50-2.25$ & 0.884 \\
Dyslipidemia & 1.59 & $0.95-2.67$ & 0.077 \\
\hline Definite spasm & 1.35 & $0.86-2.14$ & 0.197 \\
\hline Atherosclerosis $>$ 50\% & 1.48 & $0.74-2.93$ & 0.267 \\
\hline Nitrates & 1.99 & $1.23-3.20$ & 0.005 \\
\hline 1st generation CCBs & Reference & \\
\hline 2nd generation CCBs & 0.63 & $0.28-1.38$ & 0.246 \\
\hline
\end{tabular}

HR, hazard ratio; CCB, calcium channel blocker; CI, confidence interval; CHD, coronary heart disease; HTN, hypertension; DM, diabetes mellitus.

\section{Patients with definite spasm}

We analyzed the patients with definite spasm. The rate of the primary endpoint was marginally higher in the nifedipine group as compared to the diltiazem group, $13.46 \%$ vs. $5.85 \%$ respectively and the person-month incidence rate was higher in the nifedipine group as compared to the diltiazem group, 5.22 vs. 2.38 respectively (HR, 2.30; 95\% CI, 1.00 to 5.25; $p=0.049$ ) (Supplementary Table 5). The individual endpoints were not statistically significantly different among the four groups (Supplementary Table 5).

The patients treated with nifedipine and benidipine had significantly better controlled angina symptoms, especially in patients with definite spasm at the 1st to 3rd year follow-up as compared to diltiazem group (Supplementary Table 6). Individual CCBs were not an independent risk for the primary outcome by Cox-multivariate proportional hazard model for primary endpoint in patients with definitive spasm (Supplementary Table 7). 
Table 4. Subgroup analysis and interaction between 1st and 2nd generation CCBs

\begin{tabular}{|c|c|c|c|c|c|}
\hline Variable & $\begin{array}{l}\text { 1st generation CCBs }{ }^{\mathrm{a}} \\
(\mathrm{n}=1,345)\end{array}$ & $\begin{array}{l}\text { 2nd generation } \mathrm{CCBs}^{\mathrm{b}} \\
(\mathrm{n}=24 \mathrm{1})\end{array}$ & $\mathrm{HR}^{\mathrm{c}}$ & $95 \%$ CI & $p$ for interaction \\
\hline Overall & $72 / 1,345(5.4)$ & $7 / 241(2.9)$ & 0.54 & $0.25-1.17$ & \\
\hline \multicolumn{6}{|l|}{ Sex } \\
\hline Male & $46 / 826(5.6)$ & $7 / 170(4 \cdot 1)$ & 0.74 & $0.33-1.64$ & \\
\hline Female & $26 / 519(5.1)$ & o/71 (0.o) & & & \\
\hline Age, yr & & & & & 0.678 \\
\hline$<65$ & $57 / 1,079(5.3)$ & $5 / 189(2.7)$ & 0.49 & $0.20-1.23$ & \\
\hline$\geq 65$ & $15 / 266(5.6)$ & $2 / 52(3.9)$ & 0.73 & $0.17-3.21$ & \\
\hline Smoking & & & & & 0.122 \\
\hline Non-smoker & $48 / 938(5.1)$ & $3 / 183(1.6)$ & 0.32 & $0.10-1.03$ & \\
\hline Current smoker & $24 / 389(6.2)$ & $4 / 57(7.0)$ & 1.11 & $0.38-3.19$ & \\
\hline Alcohol & & & & & 0.195 \\
\hline Non-drinker & $44 / 782(5.6)$ & $2 / 125(1.6)$ & 0.29 & $0.07-1.18$ & \\
\hline Drinker & $28 / 563(5.0)$ & $5 / 115(4 \cdot 3)$ & 0.87 & $0.34-2.25$ & \\
\hline HTN & & & & & 0.885 \\
\hline No & $49 / 864(5 \cdot 7)$ & $4 / 120(3 \cdot 3)$ & 0.59 & $0.21-1.63$ & \\
\hline Yes & $23 / 480(4.8)$ & $3 / 121(2.5)$ & 0.51 & $0.15^{-1.71}$ & \\
\hline $\mathrm{DM}$ & & & & & 0.519 \\
\hline No & $65 / 1,220(5 \cdot 3)$ & $6 / 223(2.7)$ & 0.50 & $0.22-1.16$ & \\
\hline Yes & $7 / 124(5 \cdot 7)$ & $1 / 17(5.9)$ & 1.07 & $0.13-8.72$ & \\
\hline Dyslipidemia & & & & & 0.208 \\
\hline No & $55 / 1,110(5.0)$ & $4 / 199(2.0)$ & 0.40 & $0.15-1.10$ & \\
\hline Yes & $17 / 233(7 \cdot 3)$ & $3 / 43(7 \cdot 3)$ & 1.09 & $0.32-3.76$ & \\
\hline ACS before diagnosis & & & & & - \\
\hline No & $68 / 1,315(5 \cdot 2)$ & $7 / 236(3.0)$. & 0.21 & $0.52-0.87$ & \\
\hline Yes & $4 / 27(14 \cdot 9)$ & o/5(o.o) & - & & \\
\hline \multicolumn{6}{|l|}{ History of CHD } \\
\hline No & $57 / 1,190(4.8)$ & $7 / 200(3.5)$ & 0.73 & $0.33-1.59$ & - \\
\hline Yes & $15 / 154(9.8)$ & $0 / 40(0.0)$ & - & & \\
\hline History of PCI & & & & & - \\
\hline No & $65 / 1,314(5 \cdot 0)$ & $7 / 237(3.0)$ & 0.60 & $0.27-1.30$ & \\
\hline Yes & $7 / 31(22.6)$ & o/4 (0.0) & - & & \\
\hline Spasm severity & & & & & 0.881 \\
\hline Intermediate & $37 / 814(4,6)$ & $4 / 15(2.7)$ & 0.58 & $0.20-1.61$ & \\
\hline Definite & $35 / 531(6.6)$ & $3 / 90(3.3)$ & 0.52 & $0.16-1.68$ & \\
\hline Atherosclerosis & & & & & - \\
\hline$<50 \%$ & $62 / 1,239(5.0)$ & $7 / 225(3.1)$ & 0.62 & $0.28-1.35$ & \\
\hline$\geq 50 \%$ & $10 / 106(9.4)$ & $0 / 16(0.0)$ & & & \\
\hline
\end{tabular}

Values are presented as the number of incidence cases of the primary outcome/number (\%).

CCB, calcium channel blocker; HR, hazard ratio; CI, confidence interval; HTN, hypertension; DM, diabetes mellitus; ACS, acute coronary syndrome; CHD, coronary heart disease; PCI, percutaneous coronary intervention.

ast generation CCBs indicate diltiazem and nifedipine.

$\mathrm{b}_{2}$ nd-generation CCBs indicate amlodipine and benidipine.

${ }^{\mathrm{c}} \mathrm{HR}$ of the patients with 2nd generation calcium channel blockers as compared to patients with 1st generation calcium channel blockers. 
Kim SE, et al. Calcium channel blockers in vasospastic angina

Table 5. Angina symptoms grade according to calcium channel blocker by ordered logistic regression

\begin{tabular}{|c|c|c|c|c|c|c|}
\hline \multirow{2}{*}{ Variable } & \multicolumn{3}{|c|}{ Unadjusted } & \multicolumn{3}{|c|}{ Adjusted } \\
\hline & OR & $95 \% \mathrm{CI}$ & $p$ value & OR & $95 \% \mathrm{CI}$ & $p$ value \\
\hline \multicolumn{7}{|l|}{ 1st year } \\
\hline Diltiazem & Reference & & & Reference & & \\
\hline Amlodipine & 1.65 & $1.09-2.48$ & 0.017 & 1.60 & $1.05-2.45$ & 0.029 \\
\hline Nifedipine & 0.73 & $0.46-1.16$ & 0.184 & 0.64 & $0.40-1.03$ & 0.069 \\
\hline Benidipine & 0.43 & $0.24-0.77$ & 0.005 & 0.43 & $0.23-0.79$ & 0.006 \\
\hline \multicolumn{7}{|l|}{ 2nd year } \\
\hline Diltiazem & Reference & & & Reference & & \\
\hline Amlodipine & 1.16 & $0.69-1.93$ & 0.581 & 1.22 & $0.71-2.09$ & 0.465 \\
\hline Nifedipine & 0.53 & $0.32-0.88$ & 0.013 & 0.48 & $0.29-0.81$ & 0.006 \\
\hline Benidipine & 0.23 & $0.11-0.46$ & 0.000 & 0.20 & $0.10-0.41$ & 0.000 \\
\hline \multicolumn{7}{|l|}{ 3rd year } \\
\hline Diltiazem & Reference & & & Reference & & \\
\hline Amlodipine & 0.66 & $0.39-1.11$ & 0.120 & 0.71 & $0.41-1.22$ & 0.211 \\
\hline Nifedipine & 0.52 & $0.32-0.85$ & 0.009 & 0.49 & $0.29-0.82$ & 0.006 \\
\hline Benidipine & 0.17 & $0.09-0.32$ & 0.000 & 0.19 & $0.10-0.37$ & 0.000 \\
\hline
\end{tabular}

Adjusted models included age, sex, smoking, hypertension, diabetes mellitus, spasm severity, degree of atherosclerosis by angiography, history of coronary artery disease.

OR, odds ratio; CI, confidence interval.

\section{DISCUSSION}

The VA-Korea is a large scale nation-wide multicenter registry including patients with a suspicion of VA who were receiving an EG provocation test with a long-term follow-up. In our comparison of the different types of CCBs, there was no difference in the composite clinical outcomes of death from any cause, ACS, and new onset symptomatic arrhythmia in two category of classification of CCBs: comparison of the 1st and 2nd generation CCBs and comparison of each of the four CCBs in each group.

However, we found a better clinical outcome in the users of 2nd generation of CCBs in regard to ACS occurrence despite of failing attainment of statistical significance in cox multivariate regression analysis. We also demonstrated the beneficial effect of benidipine in controlling the angina symptoms as compared with diltiazem which is the most widely used CCB in Korea.

CCBs inhibit the inflow of $\mathrm{Ca}^{2+}$ into the smooth muscle cell through voltage-sensitive $\mathrm{Ca}^{2}$ channels, thereby causing vasodilation. Thus, CCBs are generally recom- mended to control angina and vasospasm. Other multicenter cohort studies performed in South Korea have included 2,032 patients, the CCBs were prescribed to $98.1 \%$ of patients and diltiazem was the most prescribed drug followed by dihydropyridine drug [6]. Diltiazem is the mostly used drug for VA patients in Japan [14,17]. Our study results also support those prior studies with 90.2\% prescription rate of CCBs in VA patients and diltiazem was the most widely used CCB (79.0\%) followed by amlodipine, nifedipine, and benidipine in VA patients.

We found that and generation CCBs were the preferred choice for ACS prevention in VA patients. It is well-known that coronary artery vasospasm is one of the most important etiologies for ACS $[18,19]$. If VA patients present with ACS, the prognosis may worsen. One retrospective study in Korea showed that $15 \%$ of VA patients presented with ACS, and they had increased risk of allcause death, adverse cardiovascular events, recurrent MI, and re-hospitalization [8]. Therefore, the prevention of ACS in VA patients is of the utmost importance. In our analysis, the and generation CCBs group showed less event of ACS occurrence than those in 1st genera- 
tion CCBs. The benefit can be explained by the longer sustainability and vessel specific property of the and generation CCBs $[20,21]$. Short acting CCBs are known to increase in sympathetic activity assessed by norepinephrine levels [20], which is associated poor outcome in patients with ischemic heart disease. Thus, we recommend the and generation CCBs to prevent future ACS as well as to control angina symptom for VA patients instead of 1st generation CCBs which is mostly used in Korea.

As for the control of angina attack in patients with VA, we demonstrated the benefit of and generation CCBs, especially benidipine in better control of angina. Benidipine do not show positive results in clinical outcomes. There have been studies showing positive clinical efficacy let alone the better control of angina symptom with benidipine in VA patients $[17,22,23]$. Representative one is meta-analysis pooled with 1,997 patients which showed that benidipine had significantly better prognostic effect as compared with amlodipine, nifedipine or diltiazem [14]. Notably, the benefit of benidipine was apparent later on 6th year which might suggest that long-term vasculoprotective effects were mainly involved instead of anti-vasospastic efficacy of benidipine. Thus, the follow-up duration of our study of 3 years, was not long enough to show the clinical benefit of this drug in our study patients as well as the small sample size of benidipine group. This medication has been reported to be more selective for coronary artery smooth muscle cells and to improve vascular endothelial function assessed by flow-mediated dilation in VA patients as compared with other CCBs [21,24]. These actions could, in part, explain the better control of angina symptoms in our study [25].

When we compared the CCBs in patients with definite spasm, and generation CCBs, benidipine consistently showed better control of angina. Nifedipine also demonstrated better control of angina symptoms in definite spasm. We agree that these results could also be due to chance as there were smaller number of patients receiving benidipine and nifedipine ( 24 to 66 persons) than those receiving diltiazem. Although nifedipine showed the signal of poor composite clinical outcome, the $p$ value and CI was marginal (HR, 2.30; 95\% CI, 1.00 to 5.26; $p=0.049$ ). Therefore, this results could be attributed to small sample size and simply by chance. In addition,
Cox-multivariate regression analysis showed that use of nifedipine was not an independent risk for poor clinical outcome in this definite spasm patients (Supplementary Table 7). Thus, we think that nifedipine could be used safely and might be fit for severe definite spasm patient for angina control. Taking into account all the things together, we would like to recommend practitioners to prescribe and generation CCBs for Korean VA patients, especially those with definite spasm for preventing ACS and controlling angina.

VA patients. In our study, the use of nitrates was only a significant risk factor of primary outcome in patients with VA. Although nitrates are frequently used to control angina symptoms, there are several studies that have demonstrated a worse prognosis of patients with VA after treatment with nitrates [26]. Potential mechanisms include the rapid development of tolerance and generation of reactive oxygen species, leading to endothelial dysfunction, sympathetic nerve activation, and increased sensitivity to vasoconstrictors. Therefore, further studies are needed before we can conclude that nitrate therapy is harmful and discontinue this drug for VA patients.

Our study has limitations. Firstly, only a small number of patients received benidipine; 65 patients. Although we demonstrated that benidipine treatment is an independent risk factor by multiple regression analysis, there might be a bias contributed by the small size of the group. Secondly, due to the small sample size of the benidipine and nifedipine groups, we did not perform a propensity matching analysis to balance baseline characteristics among groups. This difference may have influenced the results in spite of other compensatory statistical methodology of multiple regression analysis and Cox-regression analysis. Thirdly, we do not have the data on drug dose and drug adherence. Furthermore, we do not present the data on changes in the drug treatment like dose increase, or adding or changing to another drug during follow-up, which could affect the outcome and symptom management. Fourth, it was a non-randomized observational study and results are prone to be influenced by inherent limitations. Thus, prospective randomized study is needed to confirm the present results. Fifth, we do not present the data on angina symptom severity and duration, which could be another marker for estimating angina control with CCBs. 
We did not include these at the initial stage of study designing. Sixth, we do not present the data on alcohol habit, which may affect the outcome and angina symptoms in VA patients.

To conclude, CCBs are widely used in Korean VA patients, of which diltiazem is the most commonly prescribed. In our comparison between the 1st and and generation CCBs, we did not find any difference in the composite primary outcome of total death, ACS, and symptomatic arrhythmia at the 3-year follow-up. However, the use of the and generation CCBs-amlodipine and benidipine, was associated with a lower rate of ACS, and the use of benidipine was associated with less angina symptom at 3-year follow-up in VA patients.

\section{KEY MESSAGE}

1. Diltiazem was the most widely used calcium channel blocker in Korea.

2. The type of calcium channel blockers was considered to have no effect on the clinical outcomes of vasospastic angina (VA) patients.

3. The second generation calcium channel blockers seem to be associated with lower rate of acute coronary syndrome in VA patients.

4. The use of benidipine significantly improved the control of angina symptoms compared to other calcium channel blockers.

\section{Conflict of interest}

No potential conflict of interest relevant to this article was reported.

\section{REFERENCES}

1. Shimokawa H, Nagasawa K, Irie T, et al. Clinical characteristics and long-term prognosis of patients with variant angina. A comparative study between western and Japanese populations. Int J Cardiol 1988;18:331-349.

2. Yoo SY, Shin DH, Jeong JI, et al. Long-term prognosis and clinical characteristics of patients with variant angina. Korean Circ J 2008;38:651-658.

3. Choi BG, Park SH, Rha SW, et al. Five-year clinical outcomes in patients with significant coronary artery spasm: a propensity score-matched analysis. Int J Cardiol 2015;184:533-539.

4. Kim YH, Her AY, Rha SW, et al. Five-year major clinical outcomes according to severity of coronary artery spasm as assessed by intracoronary acetylcholine provocation test. Arch Cardiovasc Dis 2018;111:144-154.

5. Ito A, Fukumoto Y, Shimokawa H. Changing characteristics of patients with vasospastic angina in the era of new calcium channel blockers. J Cardiovasc Pharmacol 2004;44:480-485.

6. Ahn JM, Lee KH, Yoo SY, et al. Prognosis of variant angina manifesting as aborted sudden cardiac death. J Am Coll Cardiol 2016;68:137-145.

7. Rodriguez-Manero M, Oloriz T, le Polain de Waroux JB, et al. Long-term prognosis of patients with life-threatening ventricular arrhythmias induced by coronary artery spasm. Europace 2018;20:851-858.

8. Cho SW, Park TK, Gwag HB, et al. Clinical outcomes of vasospastic angina patients presenting with acute coronary syndrome. J Am Heart Assoc 2016;5:eoo4336.

9. JCS Joint Working Group. Guidelines for diagnosis and treatment of patients with vasospastic angina (Coronary Spastic Angina) (JCS 2013). Circ J 2014;78:2779-28o1.

10. Amsterdam EA, Wenger NK, Brindis RG, et al. 2014 AHA/ ACC guideline for the management of patients with non-ST-elevation acute coronary syndromes: a report of the American College of Cardiology/American Heart Association task force on practice guidelines. J Am Coll Cardiol 2014;64:e139-e228.

11. Kimura E, Kishida H. Treatment of variant angina with drugs: a survey of 11 cardiology institutes in Japan. Circulation 1981;63:844-848.

12. Higuma T, Oikawa K, Kato T, et al. Comparison of the effects of long-acting nifedipine $\mathrm{CR}$ and diltiazem $\mathrm{R}$ in patients with vasospastic angina: Aomori coronary spastic angina study. J Cardiol 2010;56:354-360.

13. Schroeder JS, Lamb IH, Bristow MR, Ginsburg R, Hung J, McAuley BJ. Prevention of cardiovascular events in variant angina by long-term diltiazem therapy. J Am Coll Cardiol 1983;1:1507-1511.

14. Nishigaki K, Inoue Y, Yamanouchi Y, et al. Prognostic effects of calcium channel blockers in patients with vasospastic angina: a meta-analysis. Circ J 2010;74:1943-1950.

15. Chahine RA, Feldman RL, Giles TD, et al. Randomized placebo-controlled trial of amlodipine in vasospastic angina. Amlodipine Study 160 Group. J Am Coll Cardiol 
1993;21:1365-1370.

16. Shin DI, Baek SH, Her SH, et al. The 24-month prognosis of patients with positive or intermediate results in the intracoronary ergonovine provocation test. JACC Cardiovasc Interv 2015;8:914-923.

17. Fukumoto Y, Yasuda S, Ito A, Shimokawa H. Prognostic effects of benidipine in patients with vasospastic angina: comparison with diltiazem and amlodipine. J Cardiovasc Pharmacol 2008;51:253-257.

18. Wang CH, Kuo LT, Hung MJ, Cherng WJ. Coronary vasospasm as a possible cause of elevated cardiac troponin I in patients with acute coronary syndrome and insignificant coronary artery disease. Am Heart J 2002;144:275-281.

19. Ong P, Athanasiadis A, Hill S, Vogelsberg H, Voehringer M, Sechtem U. Coronary artery spasm as a frequent cause of acute coronary syndrome: The CASPAR (Coronary Artery Spasm in Patients With Acute Coronary Syndrome) Study. J Am Coll Cardiol 2008;52:523-527.

20. Grossman E, Messerli FH. Effect of calcium antagonists on plasma norepinephrine levels, heart rate, and blood pressure. Am J Cardiol 1997;80:1453-1458.

21. Miwa Y, Masai H, Shimizu M. Differential effects of calci- um-channel blockers on vascular endothelial function in patients with coronary spastic angina. Circ J 2009;73:713717.

22. Io K, Minatoguchi S, Nishigaki K, et al. Effects of benidipine and some other calcium channel blockers on the prognosis of patients with vasospastic angina. Cohort study with evaluation of the ergonovine coronary spasm induction test. Arzneimittelforschung 2007;57:573-581.

23. Minatoguchi S. Vasospastic angina and Ca channel blockers. Curr Hypertens Rev 2013;9:219-223.

24. Karasawa A, Kubo K. Calcium antagonistic effects and the in vitro duration of actions of KW-3049, a new 1,4-dihydropyridine derivative, in isolated canine coronary arteries. Jpn J Pharmacol 1988;47:35-44.

25. Suzuki H, Yokoyama K, Akimoto Y, Daida H. Clinical efficacy of benidipine for vasospastic angina pectoris. Arzneimittelforschung 2007;57:20-25.

26. Park T, Park JY, Rha SW, et al. Impact of diltiazem alone versus diltiazem with nitrate on five-year clinical outcomes in patients with significant coronary artery spasm. Yonsei Med J 2017;58:90-98. 
Kim SE, et al. Calcium channel blockers in vasospastic angina

Supplementary Table 1. General and clinical characteristics of the study population

\begin{tabular}{|c|c|c|c|c|c|}
\hline Characteristic & Diltiazem $(n=1,253)$ & Amlodipine $(n=176)$ & Nifedipine $(n=92)$ & Benidipine $(n=65)$ & $p$ value $^{\mathrm{a}}$ \\
\hline Age, yr & $54.93 \pm 11.15$ & $57.03 \pm 10.80$ & $54.84 \pm 10.26$ & $53.66 \pm 10.11$ & 0.076 \\
\hline $\mathrm{BMI}, \mathrm{kg} / \mathrm{m}^{2}$ & $29.38 \pm 101.12$ & $24.96 \pm 3.18$ & $24.62 \pm 3.29$ & $24.65 \pm 2.99$ & 0.882 \\
\hline $\mathrm{DBP}, \mathrm{mmHg}$ & $127.19 \pm 36.04$ & $130.86 \pm 18.96$ & $125.88 \pm 18.55$ & $124.02 \pm 15.78$ & 0.208 \\
\hline $\mathrm{SBP}, \mathrm{mmHg}$ & $76.96 \pm 12.39$ & $78.68 \pm 12.39$ & $78.79 \pm 12.11$ & $76.80 \pm 10.96$ & 0.417 \\
\hline $\mathrm{TC}, \mathrm{mg} / \mathrm{dL}$ & $175.73 \pm 36.05$ & $171.09 \pm 37.97$ & $173.68 \pm 34.50$ & $177.67 \pm 41.21$ & 0.446 \\
\hline $\mathrm{TG}, \mathrm{mg} / \mathrm{dL}$ & $143.80 \pm 103.25$ & $150.92 \pm 104.90$ & $144.38 \pm 124.88$ & $133.11 \pm 108.10$ & 0.738 \\
\hline HDL-C, mg/dL & $46.83 \pm 13.11$ & $45 \cdot 57 \pm 10.72$ & $46.69 \pm 11.21$ & $49.15 \pm 12.51$ & 0.342 \\
\hline LDL-C, mg/dL & $104.98 \pm 31.52$ & $99.19 \pm 32.75$ & $101.03 \pm 31.81$ & $106.16 \pm 33.45$ & 0.151 \\
\hline hsCRP, mg/dL & $1.11 \pm 8.22$ & $0.42 \pm 0.92$ & $0.30 \pm 0.63$ & $0.18 \pm 0.37$ & 0.530 \\
\hline $\mathrm{CKMB}, \mathrm{ng} / \mathrm{mL}$ & $6.31 \pm 27.07$ & $5.19 \pm 12.61$ & $9.78 \pm 22.31$ & $2.19 \pm 1.81$ & 0.426 \\
\hline Troponin-I, ng/mL & $0.62 \pm 5.06$ & $0.33 \pm 2.01$ & $1.24 \pm 4.80$ & $0.03 \pm 0.10$ & 0.596 \\
\hline $\mathrm{LVEF}, \%$ & $64.50 \pm 6.18$ & $64.58 \pm 6.53$ & $65.68 \pm 7.27$ & $62.58 \pm 8.44$ & 0.041 \\
\hline Male sex & $767(61.21)$ & $123(69.89)$ & $59(64.13)$ & $47(72.31)$ & 0.052 \\
\hline Smoking & $355(28.74)$ & $46(26.29)$ & $34(36.96)$ & $11(16.92)$ & 0.047 \\
\hline HTN & $436(34.82)$ & $97(55.11)$ & $44(47.83)$ & $24(36.92)$ & 0.000 \\
\hline $\mathrm{DM}$ & $115(9.19)$ & $14(8)$ & $9(9.78)$ & $3(4.62)$ & 0.602 \\
\hline Dyslipidemia & $213(17.03)$ & $32(18.29)$ & $20(21.74)$ & $9(13.85)$ & 0.574 \\
\hline CHD & $137(10.94)$ & $27(15 \cdot 43)$ & $17(18.48)$ & $13(20)$ & 0.014 \\
\hline PCI & $29(2.31)$ & $3(1.7)$ & $2(2.17)$ & $1(1.54)$ & 0.939 \\
\hline Definite spasm & $479(38.23)$ & $66(37.5)$ & $52(56.52)$ & $24(36.92)$ & 0.006 \\
\hline Atherosclerosis $>50 \%$ & $100(7.98)$ & $15(8.52)$ & $6(6.52)$ & $1(1.54)$ & 0.266 \\
\hline
\end{tabular}

Values are presented as mean \pm SD or number $(\%)$.

CCB, calcium channel blocker; BMI, body mass index; DBP, diastolic blood pressure; SBP, systolic blood pressure; TC, total cholesterol; TG, triglyceride; HDL-C, high density lipoprotein cholesterol; LDL-C, low density lipoprotein cholesterol; hsCRP, high-sensitive C-reactive protein; CKMB, creatinine kinase-MB; LVEF, left ventricular ejection fraction; HTN, hypertension; DM, diabetes mellitus; CHD, coronary heart disease; PCI, percutaneous coronary intervention.

${ }^{a} p$ values were calculated by analysis of variance for continuous variables and calculated by chi-square for categorical variables. 
Supplementary Table 2. Incidence rates and HRs for primary composite outcome and each outcome

\begin{tabular}{|c|c|c|c|c|c|c|c|c|c|}
\hline Variable & No. & Event (\%) & Person-month & Incidence rate & $95 \% \mathrm{CI}$ & $p$ value $^{a}$ & $\mathrm{HR}^{\mathrm{b}}$ & $95 \% \mathrm{CI}$ & $p$ value $^{b}$ \\
\hline \multicolumn{6}{|c|}{ Composite outcome } & 0.291 & & & \\
\hline Diltiazem & 1,253 & $65(5.2)$ & 28,271 & 2.30 & $1.80-2.93$ & & Reference & & \\
\hline Amlodipine & 176 & $4(2.3)$ & 3,666 & 1.09 & $0.41-2.91$ & & 0.44 & $0.16-1.22$ & 0.115 \\
\hline Nifedipine & 92 & $7(7.6)$ & 2,571 & 2.72 & $1.30-5.71$ & & 1.39 & $0.64-3.04$ & 0.406 \\
\hline Benidipine & 65 & $3(4 \cdot 6)$ & 1,904 & 1.58 & $0.51-4.89$ & & 0.83 & $0.26-2.66$ & 0.759 \\
\hline Arrhythymia & & & & & & 0.763 & & & \\
\hline Diltiazem & 1,253 & $17(1.4)$ & 29,764 & 0.57 & $0.36-0.92$ & & Reference & & \\
\hline Amlodipine & 176 & $2(1.1)$ & 3,701 & 0.54 & $0.14-2.16$ & & 0.92 & $0.21-3.96$ & 0.906 \\
\hline Nifedipine & 92 & $2(2.2)$ & 2,719 & 0.74 & $0.18-2.94$ & & 1.38 & $0.32-5.96$ & 0.670 \\
\hline Benidipine & 65 & $2(3.1)$ & 1,938 & 1.03 & $0.26-4.13$ & & 2.07 & $0.48-8.95$ & 0.331 \\
\hline ACS & & & & & & 0.134 & & & \\
\hline Diltiazem & 1,253 & $49(3.9)$ & 29,254 & 1.68 & $1.27-2.22$ & & Reference & & \\
\hline Amlodipine & 176 & 1 (0.6) & 3,712 & 0.27 & $0.04-1.91$ & & 0.16 & $0.02-1.17$ & 0.071 \\
\hline Nifedipine & 92 & $4(4 \cdot 4)$ & 2,728 & 1.47 & $0.55^{-3.91}$ & & 0.90 & $0.32-2.48$ & 0.833 \\
\hline Benidipine & 65 & $1(1.5)$ & 1,989 & 0.50 & $0.07-3 \cdot 57$ & & 0.32 & $0.04-2.33$ & 0.261 \\
\hline Death & & & & & & 0.525 & & & \\
\hline Diltiazem & 1,253 & $3(0.2)$ & 30,094 & 0.10 & $0.03-0.31$ & & Reference & & \\
\hline Amlodipine & 176 & $1(0.6)$ & 3,731 & 0.27 & $0.04-1.90$ & & 2.62 & $0.27-25.25$ & 0.404 \\
\hline Nifedipine & 92 & $1(1.1)$ & 2,779 & 0.36 & $0.05-2.55$ & & 3.64 & $0.38-35.11$ & 0.264 \\
\hline Benidipine & 65 & 0 & 2,011 & 0.00 & & & NA & & \\
\hline
\end{tabular}

HR, hazard ratio; CI, confidence interval; ACS, acute coronary syndrome; NA, not applicable.

${ }^{a} p$ value by log rank test.

${ }^{\mathrm{b}} \mathrm{p}$ value by univariable Cox proportional hazard regression. 
Supplementary Table 3. HRs for the primary composite outcome by Cox regression

\begin{tabular}{|c|c|c|c|}
\hline Variable & HR & $95 \% \mathrm{CI}$ & $p$ value \\
\hline Age & 1.00 & $0.98-1.02$ & 0.876 \\
\hline Female sex & 0.94 & $0.55^{-1.60}$ & 0.823 \\
\hline History of CHD & 1.71 & $0.96-3.04$ & 0.069 \\
\hline Smoking & 1.15 & $0.68-1.95$ & 0.602 \\
\hline Hypertension & 0.77 & $0.47-1.26$ & 0.295 \\
\hline $\mathrm{DM}$ & 1.06 & $0.50-2.26$ & 0.875 \\
\hline Dyslipidemia & 1.58 & $0.94-2.65$ & 0.082 \\
\hline Definite spasm & 1.31 & $0.83-2.07$ & 0.252 \\
\hline Atherosclerosis $>50 \%$ & 1.50 & $0.75-2.98$ & 0.252 \\
\hline Nitrates & 1.98 & $1.23-3.20$ & 0.005 \\
\hline \multicolumn{4}{|l|}{ Calcium channel blocker } \\
\hline Diltiazem & Reference & & \\
\hline Amlodipine & 0.49 & $0.18-1.36$ & 0.171 \\
\hline Nifedipine & 1.28 & $0.58-2.81$ & 0.542 \\
\hline Benidipine & 1.01 & $0.31-3.28$ & 0.986 \\
\hline
\end{tabular}

HR, hazard ratio; CI, confidence interval; CHD, coronary heart disease; DM, diabetes mellitus. 
KJIM'

Supplementary Table 4. ORs for the primary composite outcome and re-admission/emergency room visit by logistic regression analysis

\begin{tabular}{|c|c|c|c|}
\hline Variable & OR & $95 \% \mathrm{CI}$ & $p$ value \\
\hline Age & 1.00 & $0.98-1.01$ & 0.664 \\
\hline Female sex & 0.95 & $0.69-1.30$ & 0.752 \\
\hline History of CHD & 1.81 & $1.25-2.62$ & 0.002 \\
\hline Smoking & 1.00 & $0.71-1.39$ & 0.980 \\
\hline Hypertension & 1.03 & $0.77-1.38$ & 0.855 \\
\hline $\mathrm{DM}$ & 0.85 & $0.51-1.40$ & 0.513 \\
\hline Dyslipidemia & 1.24 & $0.88-1.75$ & 0.225 \\
\hline Definite spasm & 0.88 & $0.66-1.18$ & 0.395 \\
\hline Atherosclerosis $\geq 50 \%$ & 1.61 & $1.02-2.56$ & 0.041 \\
\hline Nitrates & 1.10 & $0.78-1.55$ & 0.576 \\
\hline \multicolumn{4}{|l|}{ Calcium channel blocker } \\
\hline Diltiazem & Reference & & \\
\hline Amlodipine & 0.93 & $0.60-1.46$ & 0.759 \\
\hline Nifedipine & 1.33 & $0.77-2.27$ & 0.304 \\
\hline Benidipine & 1.30 & $0.68-2.46$ & 0.427 \\
\hline
\end{tabular}

OR, odds ratio; CI, confidence interval; CHD, coronary heart disease; DM, diabetes mellitus. 
Kim SE, et al. Calcium channel blockers in vasospastic angina

Supplementary Table 5 . Incidence rates and HR for primary composite outcome and each outcome among patients with definite spasm

\begin{tabular}{|c|c|c|c|c|c|c|c|c|c|}
\hline Variable & No. & Event (\%) & $\begin{array}{l}\text { Person- } \\
\text { month }\end{array}$ & $\begin{array}{c}\text { Incidence } \\
\text { rate }\end{array}$ & $95 \% \mathrm{CI}$ & $p$ value $^{a}$ & $\mathrm{HR}^{\mathrm{b}}$ & $95 \% \mathrm{CI}$ & $p$ value $^{b}$ \\
\hline Composite outcome & & & & & & 0.1218 & & & \\
\hline Diltiazem & 479 & $28(5.85)$ & 11,747 & 2.38 & $1.65-3.45$ & & Reference & & \\
\hline Amlodipine & 66 & $2(3.0)$ & 1,268 & 1.58 & $0.39-6.31$ & & 0.55 & $0.13-2.30$ & 0.411 \\
\hline Nifedipine & 52 & $7(13.46)$ & 1,341 & 5.22 & $2.49-10.95$ & & 2.30 & $1.00-5.26$ & 0.049 \\
\hline Benidipine & 24 & $1(4 \cdot 17)$ & 895 & 1.12 & $0.16-7.93$ & & 0.67 & $0.09-4.93$ & 0.695 \\
\hline Arrhythmia & & & & & & 0.5591 & & & \\
\hline Diltiazem & 479 & $10(2.09)$ & 12,335 & 0.81 & $0.44^{-1.51}$ & & Reference & & \\
\hline Amlodipine & 66 & o & 1,303 & 0.00 & - & & - & & \\
\hline Nifedipine & 52 & $2(3.85)$ & 1,489 & 1.34 & $0.34-5 \cdot 37$ & & 1.73 & $0.38-7.91$ & 0.478 \\
\hline Benidipine & 24 & $1(4.17)$ & 895 & 1.12 & $0.16-7.93$ & & 1.78 & $0.23-13.95$ & 0.581 \\
\hline ACS & & & & & & 0.3465 & & & \\
\hline Diltiazem & 479 & $20(4.18)$ & 12,216 & 1.64 & $1.06-2.54$ & & Reference & & \\
\hline Amlodipine & 66 & $1(1.52)$ & 1,307 & 0.77 & $0.11-5.43$ & & 0.46 & $0.06-3.44$ & 0.451 \\
\hline Nifedipine & 52 & $4(7.69)$ & 1,498 & 2.67 & $1.00-7.12$ & & 1.61 & $0.55-4.73$ & 0.383 \\
\hline Benidipine & 24 & o & 948 & 0.00 & - & & - & & \\
\hline Death & & & & & & 0.4358 & & & \\
\hline Diltiazem & 479 & $2(0.42)$ & 12,549 & 0.16 & $0.04-0.64$ & & Reference & & \\
\hline Amlodipine & 66 & $1(1.52)$ & 1,326 & 0.75 & $0.11-5.35$ & & 4.17 & $0.38-46.02$ & 0.244 \\
\hline Nifedipine & $5^{2}$ & $1(1.92)$ & 1,549 & 0.65 & $0.09-4.58$ & & 4.22 & $0.38-46.55$ & 0.240 \\
\hline Benidipine & 24 & 0 & 948 & 0.00 & - & & - & & \\
\hline
\end{tabular}

HR, hazard ratio; CI, confidence interval; ACS, acute coronary syndrome.

ap value by log rank test.

${ }^{\mathrm{b}} \mathrm{p}$ value by univariate Cox proportional hazard regression. 
Supplementary Table 6. Angina symptoms grade and calcium channel blocker by ordered logistic regression in patients with definite spasm

\begin{tabular}{|c|c|c|c|c|c|c|}
\hline \multirow{2}{*}{ Variable } & \multicolumn{3}{|c|}{ Unadjusted } & \multicolumn{3}{|c|}{ Adjusted } \\
\hline & OR & $95 \% \mathrm{CI}$ & $p$ value & OR & $95 \% \mathrm{CI}$ & $p$ value \\
\hline \multicolumn{7}{|l|}{ 1st year } \\
\hline Diltiazem & Reference & & & Reference & & \\
\hline Amlodipine & 1.33 & $0.71-2.49$ & 0.367 & 1.13 & $0.59-2.16$ & 0.718 \\
\hline Nifedipine & 0.49 & $0.27-0.90$ & 0.021 & 0.38 & $0.20-0.72$ & 0.003 \\
\hline Benidipine & 0.64 & $0.25-1.62$ & 0.349 & 0.53 & $0.20-1.38$ & 0.194 \\
\hline \multicolumn{7}{|l|}{ 2nd year } \\
\hline Diltiazem & Reference & & & Reference & & \\
\hline Amlodipine & 1.46 & $0.65-3.29$ & 0.364 & 1.17 & $0.50-2.75$ & 0.711 \\
\hline Nifedipine & 0.34 & $0.18-0.65$ & 0.001 & 0.29 & $0.15-0.56$ & 0.000 \\
\hline Benidipine & 0.28 & $0.11-0.74$ & 0.010 & 0.20 & $0.07-0.55$ & 0.002 \\
\hline \multicolumn{7}{|l|}{ 3rd year } \\
\hline Diltiazem & Reference & & & Reference & & \\
\hline Amlodipine & 0.85 & $0.35-2.04$ & 0.712 & 0.78 & $0.31-1.94$ & 0.589 \\
\hline Nifedipine & 0.35 & $0.18-0.66$ & 0.001 & 0.27 & $0.14-0.54$ & 0.000 \\
\hline Benidipine & 0.15 & $0.06-0.39$ & 0.000 & 0.13 & $0.05-0.36$ & 0.000 \\
\hline
\end{tabular}

Adjusted models included age, sex, smoking, hypetension, diabetes mellitus, spasm severity, degree of atherosclerosis by angiography, history of coronary heart disease.

OR, odds ratio; CI, confidence interval. 
Kim SE, et al. Calcium channel blockers in vasospastic angina

Supplementary Table 7. HR for the primary composite outcome by Cox regression among patients with definite spasm

\begin{tabular}{|c|c|c|c|}
\hline Variable & $\mathrm{HR}$ & $95 \% \mathrm{CI}$ & $p$ value \\
\hline Age & 1.01 & $0.98-1.04$ & 0.607 \\
\hline Female sex & 1.23 & $0.57-2.65$ & 0.604 \\
\hline History of CHD & 2.40 & $1.16-4.94$ & 0.018 \\
\hline Smoking & 1.43 & $0.66-3.11$ & 0.368 \\
\hline Alcohol drinking & 0.73 & $0.35^{-1.54}$ & 0.412 \\
\hline Hypertension & 0.82 & $0.42-1.62$ & 0.571 \\
\hline $\mathrm{DM}$ & 0.44 & $0.10-1.88$ & 0.269 \\
\hline Dyslipidemia & 1.56 & $0.70-3.46$ & 0.279 \\
\hline Atherosclerosis $>50 \%$ & 1.55 & $0.63-3.81$ & 0.342 \\
\hline Nitrates & 1.44 & $0.70-2.96$ & 0.319 \\
\hline Diltiazem & Reference & - & - \\
\hline Amlodipine & 0.55 & $0.13-2.36$ & 0.421 \\
\hline Nifedipine & 2.29 & $0.99-5.29$ & 0.053 \\
\hline Benidipine & 0.73 & $0.10-5.54$ & 0.761 \\
\hline
\end{tabular}

HR, hazard ratio; CI, confidence interval; CHD, coronary heart disease; DM, diabetes mellitus. 